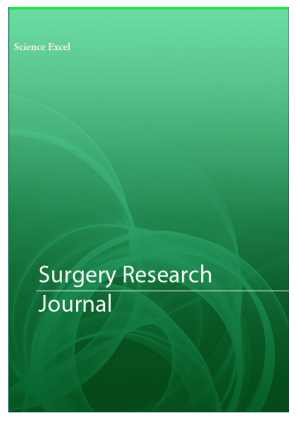

Correspondence

Adam Lewis

5338 NE 36th Street, Kansas City MO, 64117, USA.

E-mail: adamlewis@kcumb.edu

- Received Date: 24 Nov 2020

- Accepted Date: 07 Dec 2020

- Publication Date: 12 Dec 2020

Keywords

anterior segment blood supply; anterio segment ischemia; complications of strabismus surgery; eye vasculature

Copyright

(c) 2021 Science Excel. This is an openaccess article distributed under the terms of the Creative Commons Attribution 4.0 International license.

\title{
Variations in anterior segment vasculature of the eye and their implication in strabismus surgery and other surgical procedures
}

\author{
Adam Lewis ${ }^{1}$, Clayton Marolt ${ }^{1}$, M Scott Draper ${ }^{1}$, Victoria Gordon' ${ }^{1}$, Kenneth \\ Johnson' , Joanna Rowe ${ }^{1}$, Kanwalgeet Hans' , Steven M Silverstein², and \\ Anthony Olinger ${ }^{1}$
}

'Kansas City University of Medicine and Biosciences, Kansas City, Missouri, USA

${ }^{2}$ Ophthalmologist, Kansas City, Missouri, USA

\begin{abstract}
Objective: Anterior segment ischemia (ASI) is a serious complication of strabismus surgery and other ocular procedures. The purpose of this study is to evaluate variation of the long posterior artery (LPCA). These variations may predispose patients to anterior segment ischemia following strabismus surgery, and proper mapping may provide surgical "danger zones" for other invasive ocular procedures.

Subjects: A total of 100 preserved cadaveric eyeballs from University of Nebraska Medical Center (UNMC), Creighton University, and Kansas City University (KCU) were utilized for this study.

Methods: LPCAs were identified, isolated, and counted prior to removal of the choroid. Two distances were measured from within the orbit: (1) the penetration point of the LPCA through the sclera to the center of the optic nerve and (2) the penetration point of the LPCA through the sclera to the superior border of the corresponding rectus muscle. The data was analyzed to determine the number and location of LPCAs that contribute to the major arterial circle of iris. Independent sample t-tests were used to determine significant differences comparing the number of arteries and penetration points between right and left eyes, medially and laterally located arteries, and sex.

Results: Significant differences were found between subjects with one vs two LPCAs, males vs females, and medially vs laterally located arteries in at least one of the two measurements. Thirty-three of 100 eyeballs had only one LPCA (33\%).

Conclusions: This study demonstrates that a larger percentage of patients may be predisposed to anterior segment ischemia than previously reported. It also provides a surgical "danger zone" that will enable surgeons to avoid damaging the long posterior ciliary artery during invasive choroidal and orbital procedures. This knowledge will facilitate more appropriate management of patients including preoperative screenings, intraoperative isolation of the long posterior ciliary artery, more frequent postoperative monitoring, and accelerated initiation of treatment plans.
\end{abstract}

\section{Introduction}

Anterior segment ischemia (ASI) is a result of decreased blood flow to the anterior segment of the eye and has been observed in patients with occlusive vascular disorders, such as carotid artery or ophthalmic artery obstruction, leukemia, sickle cell disease, and others [1]. ASI is also a rare but serious complication of strabismus surgery resulting in irreparable vision loss [2].

There are typically seven anterior ciliary arteries which branch from the muscular arteries and travel with the extraocular musculature, two anterior ciliary arteries on each of the four rectus muscles, except for the lateral rectus muscle that is classically described as only having one. The anterior ciliary arteries supply the rectus muscles, conjunctiva, and sclera before anastomosing with the long posterior ciliary arteries to form the major arterial circle of the iris $[3,4]$. There are 1-2 long posterior ciliary arteries that travel near the optic nerve and pierce the posterior aspect of the sclera to supply the choroid and ciliary muscle before joining the major arterial circle of the iris [4]. This anastomosis of the anterior ciliary arteries with the long posterior ciliary arteries in the major arterial circle of iris is vital in maintaining perfusion during times of decreased blood flow such as strabismus surgery [5].

ASI occurs when the anterior ciliary artery is severed along its course through the extraocular muscles during disinsertion from the sclera as part of the procedure. Interruption of blood flow through the anterior ciliary vessels leads to a decrease in blood supply to the anterior segment and may result in ischemia if the long posterior ciliary arteries are unable to provide adequate collateral circulation. The communication and integrity of these arteries and their anatomic patterns is vital in prevention of ASI [5]. Although variations and mapping of the long posterior ciliary artery 
have not been extensively studied, anterior segment vessel density, occlusion, and variations to the vascular network of the iris have given some insight into possible predispositions to anterior segment ischemia [6-8]. Barabino \& Dana describe the long posterior ciliary artery as the most important vessel which provides blood to the anterior segment [9].

Given the importance of this relationship between the anterior and posterior ciliary arteries, this study was designed to evaluate variation of the long posterior artery and elucidate its path as it pierces the posterior sclera and travels to the major arterial circle of iris. These variations may predispose patients to anterior segment ischemia following strabismus surgery, and proper mapping may provide surgical "danger zones" for other invasive ocular procedures.

\section{Method}

A total of 100 preserved cadaveric eyeballs from University of Nebraska Medical Center (UNMC), Creighton University, and the anatomy labs on both campuses of Kansas City University (KCU) were utilized for this study. IRB/Ethics Committee ruled that approval was not required for this study. The dissection was performed via an anterior approach following removal of the orbital contents. Dissection was performed and photographs taken using a Zeiss Meditech AG dissection scope. Bulbar conjunctiva was removed, and eyelids resected to expose the insertion points of the four rectus muscles on the sclera. Each rectus muscle was isolated and cleaned to be used as a landmark for measurement. The choroid was exposed by making a shallow, circumferential incision in the sclera $1-2 \mathrm{~cm}$ from the limbus, followed by removal of overlying sclera to a point $1 \mathrm{~cm}$ proximal to the insertion point of the four rectus muscles.

Long posterior ciliary arteries were identified, isolated, and counted prior to removal of the choroid. Two distances were measured from within the orbit: (1) the penetration point of the LPCA through the sclera to the center of the optic nerve and (2) the penetration point of the LPCA through the sclera to the superior border of the corresponding rectus muscle. (Figure 1).

The data was analyzed to determine the number and location of long posterior ciliary arteries that contribute to the major arterial circle of iris. Independent sample t-tests were used to determine significant differences comparing the number of arteries and penetration points between right and left eyes, medially and laterally located arteries, and sex. The intraclass correlation coefficient (ICC) was calculated from a sample of 8 eyeballs in order to determine interrater reliability between 2 raters for all measurement points [10]. (Table 1).

Table 1. Interrater Reliability

\begin{tabular}{|l|c|c|}
\hline Measurement & $\begin{array}{l}\text { Intraclass Correlation } \\
\text { Coefficient }\end{array}$ & $\begin{array}{l}\text { Strength as according } \\
\text { to Fleiss }^{10}\end{array}$ \\
\hline 1 & .82 & Excellent \\
\hline 2 & .74 & Excellent \\
\hline 3 & .98 & Excellent \\
\hline 4 & .68 & Fair to good \\
\hline 5 & .52 & Fair to good \\
\hline 6 & .91 & Excellent \\
\hline 7 & .88 & Excellent \\
\hline 8 & .74 & Fair to good \\
\hline
\end{tabular}

Figure 1. Depiction of the two distances measured: the penetration point of the LPCA through the sclera to the center of the optic nerve and the penetration point of the LPCA through the sclera to the superior border of the corresponding rectus muscle. Also depicted is a surgical "danger zone".

\section{Results}

The average distance of the long posterior ciliary artery from the optic nerve was $0.9122 \mathrm{~cm}$ with an average distance inferior to the superior border of the rectus muscle being 0.1286. Any measurement located inferior to the superior border of the rectus muscle was assigned a negative value and is depicted as a negative value in the graphs. This is depicted in Figure 2. There were no statistically significant differences between left and right eyeballs. Subjects with one long posterior ciliary artery showed a small but significant difference in average distance from the superior border of the rectus muscle than those with two $(0.1796 \mathrm{~cm} \mathrm{v} 0.1165 \mathrm{~cm}, \mathrm{p}=0.00252)$. This is depicted in figures 3 and 4 . The average distance from the optic nerve between those with one long posterior ciliary artery versus two was not statistically significant. Males showed larger average distance of the long posterior ciliary artery from the optic nerve $(0.9321 \mathrm{~cm} \mathrm{v} 0.8821 \mathrm{~cm}, \mathrm{p}=0.0101)$ compared to females, with no statistically significant difference between average distance from the superior border of the rectus muscle, which is depicted in figures 5 and 6 . On average, the medially located long posterior ciliary arteries penetrated the sclera more distally than laterally located arteries $(0.9303 \mathrm{~cm} \mathrm{v} 0.8752 \mathrm{~cm}$, $\mathrm{p}=0.0056)$. On average, medially located arteries were also located more inferiorly than laterally located arteries $(0.1482 \mathrm{~cm}$ $\mathrm{v} 0.0795, \mathrm{p}=0.0005$ ), which is depicted in figures 7 and 8 . Sixtyseven eyes had both medially and laterally located arteries, 29 eyes had one artery located medially, and 4 eyes had one artery located laterally.

Thirty-three of 100 eyeballs had only one long posterior ciliary artery (33\%). Of these eyes, $33(100 \%)$ penetration points were found at or below the superior border of the rectus muscle depicted in figure 3.

Figure 9 depicts a surgical "danger zone" that indicates the location with the highest probability of hitting the long posterior ciliary artery as it pierces the sclera. 


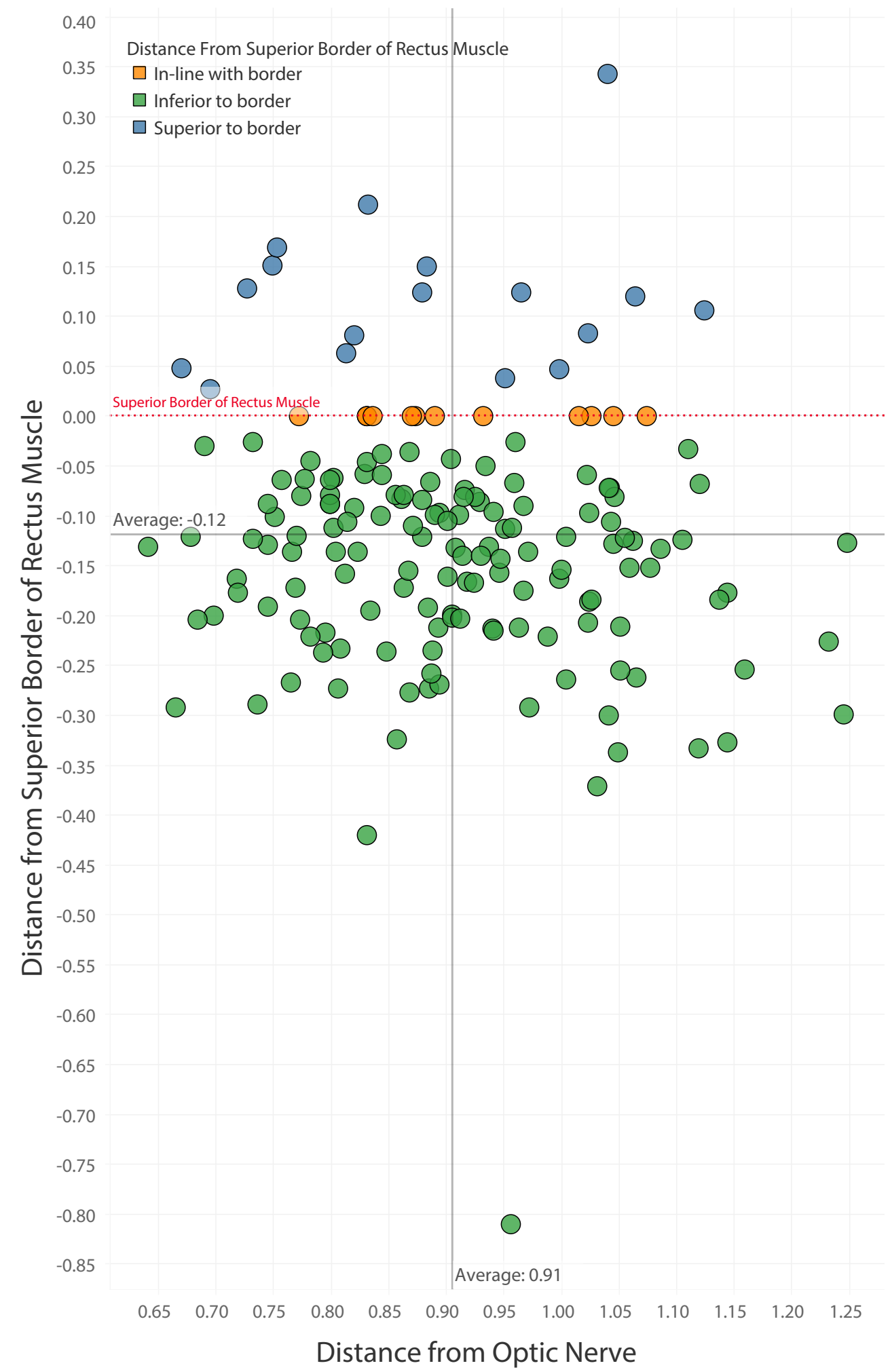

Figure 2. Penetration point of the long posterior ciliary artery. Distance from the superior border of the rectus muscle is plotted vs. the distance from the optic nerve. All data points are included $(n=167)$. 


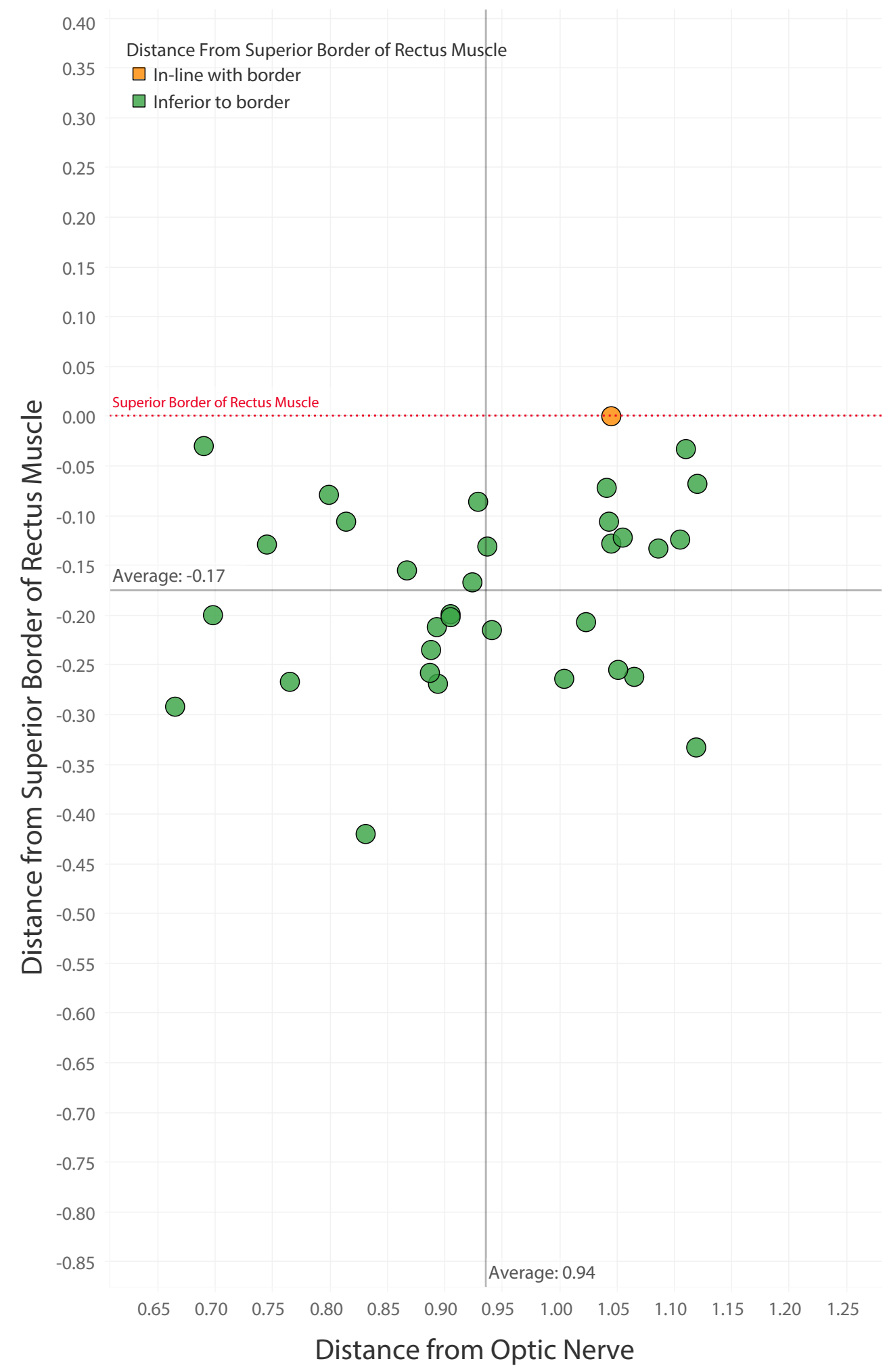

Figure 3. Penetration point of the long posterior ciliary artery. Distance from the superior border of the rectus muscle is plotted vs. the distance from the optic nerve. Data points from subjects with one artery are included $(n=33)$. 


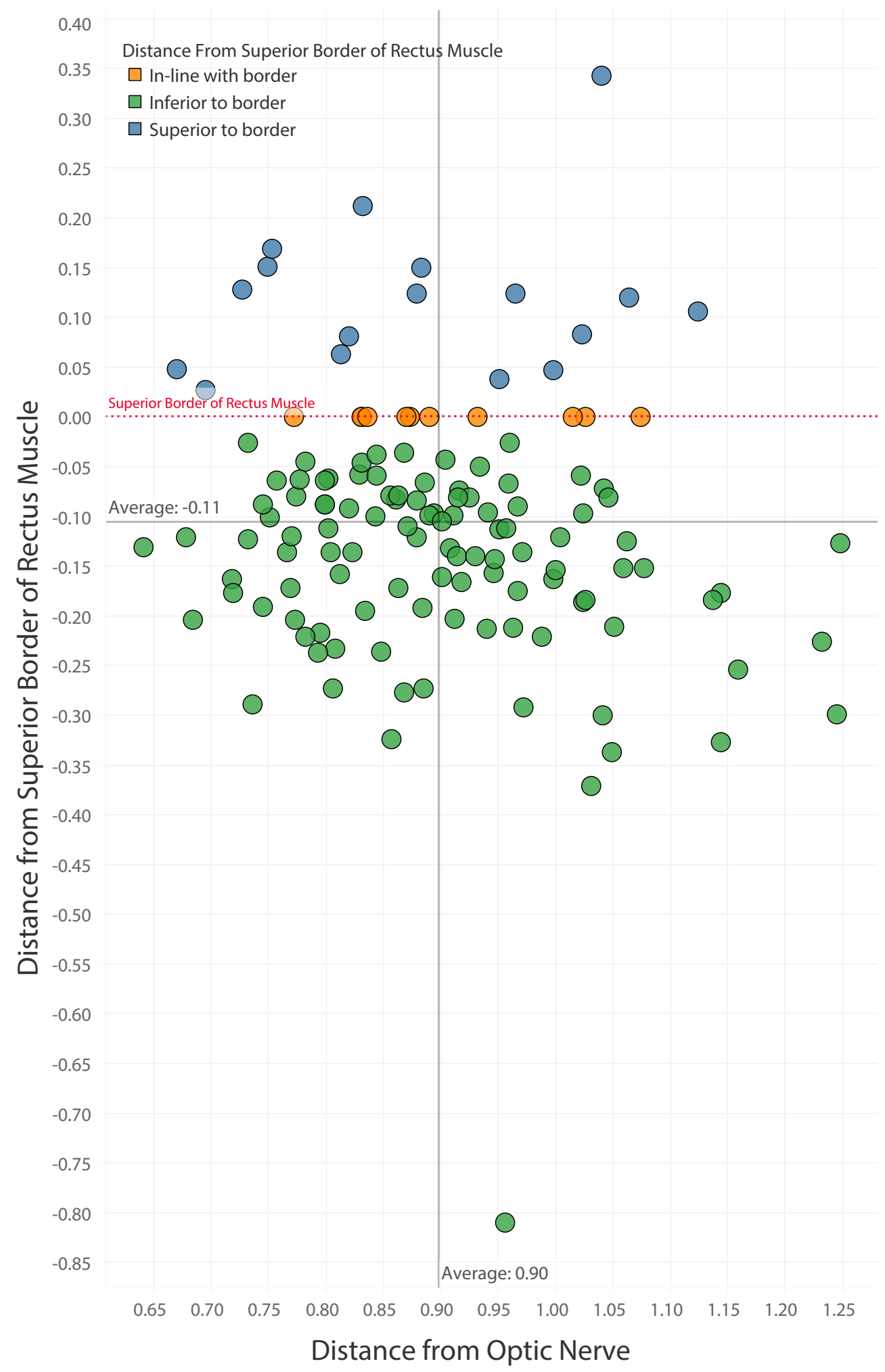

Figure 4. Penetration point of the long posterior ciliary artery. Distance from the superior border of the rectus muscle is plotted vs. the distance from the optic nerve. Data points from subjects with two arteries are included $(\mathrm{n}=134)$. 


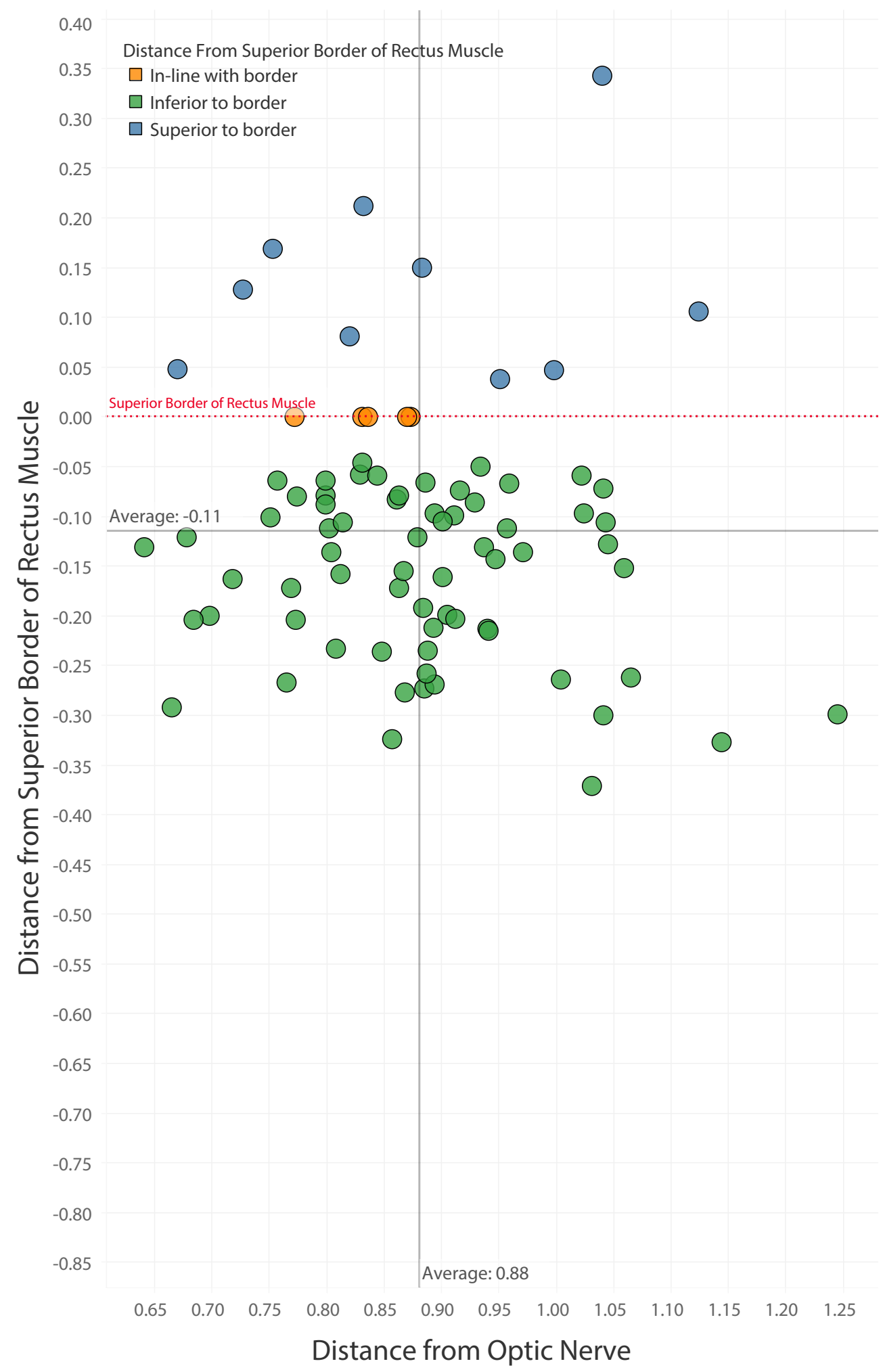

Figure 5. Penetration point of the long posterior ciliary artery. Distance from the superior border of the rectus muscle is plotted vs. the distance from the optic nerve. Data points from subjects with two arteries are included $(\mathrm{n}=134)$. 


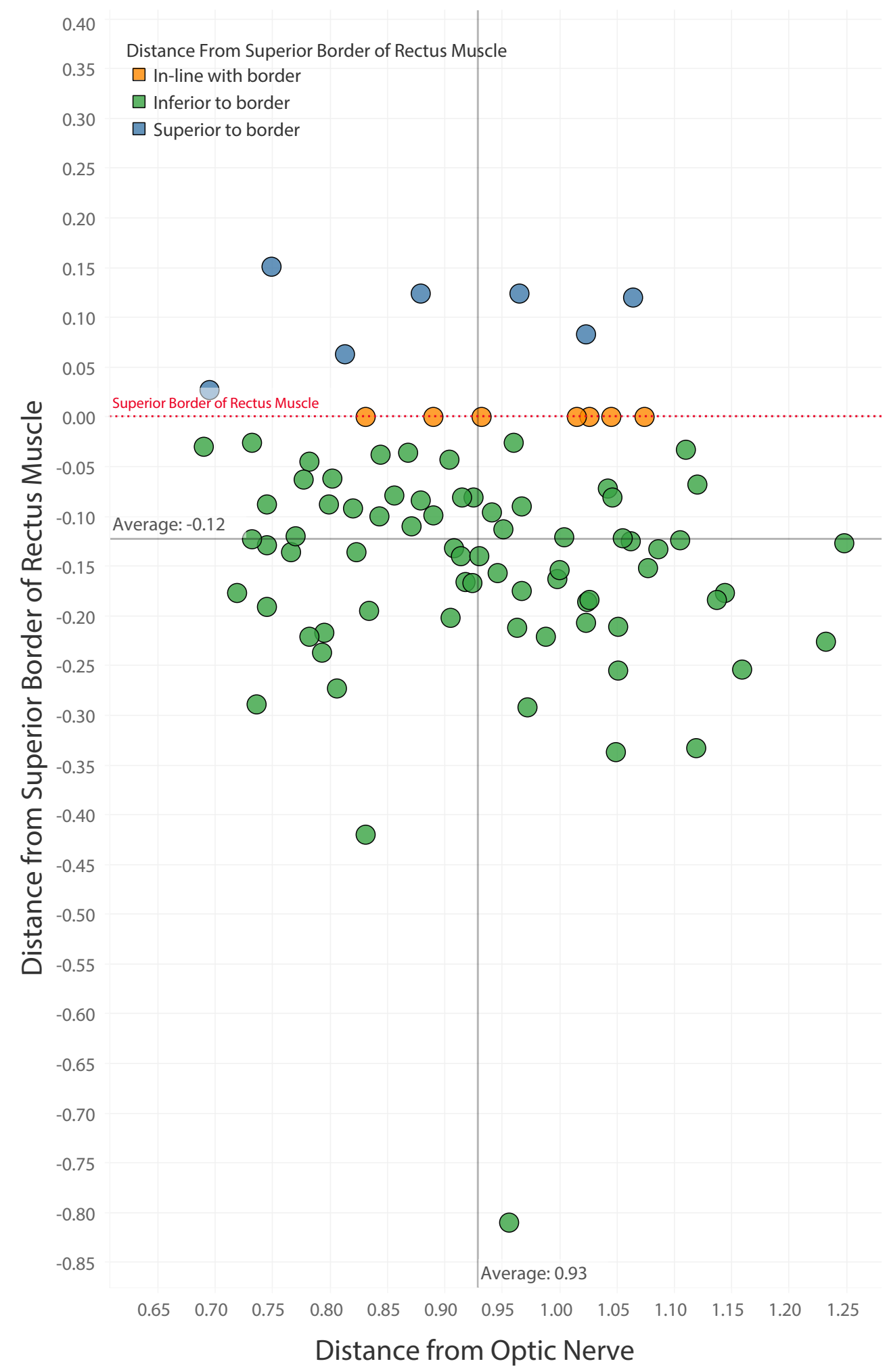

Figure 6. Penetration point of the long posterior ciliary artery. Distance from the superior border of the rectus muscle is plotted vs. the distance from the optic nerve. Data points from subjects with two arteries are included $(\mathrm{n}=134)$. 


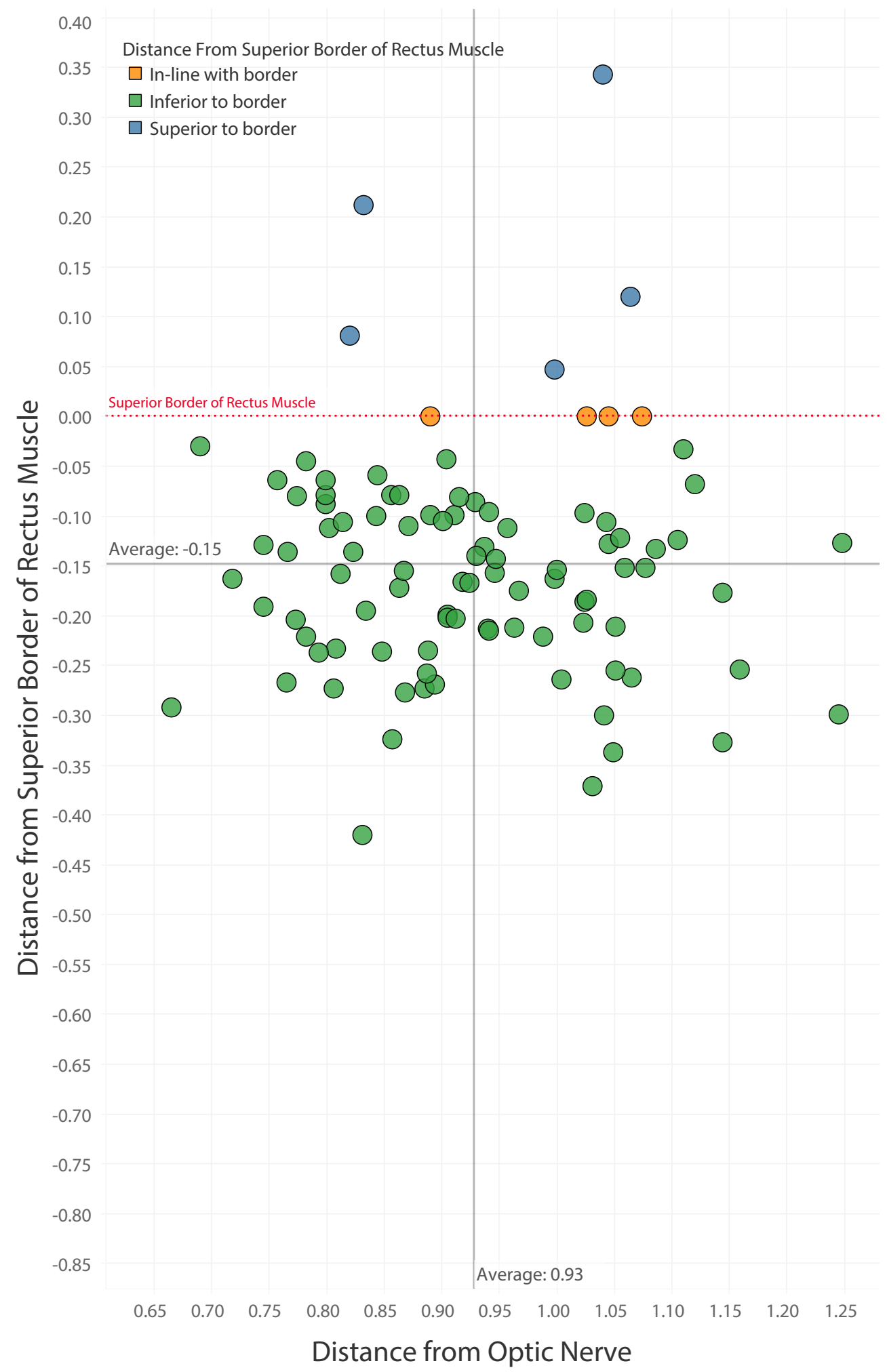

Figure 7. Penetration point of the long posterior ciliary artery. Distance from the superior border of the rectus muscle is plotted vs. the distance from the optic nerve. Data points from medially located arteries are included $(n=96)$. 


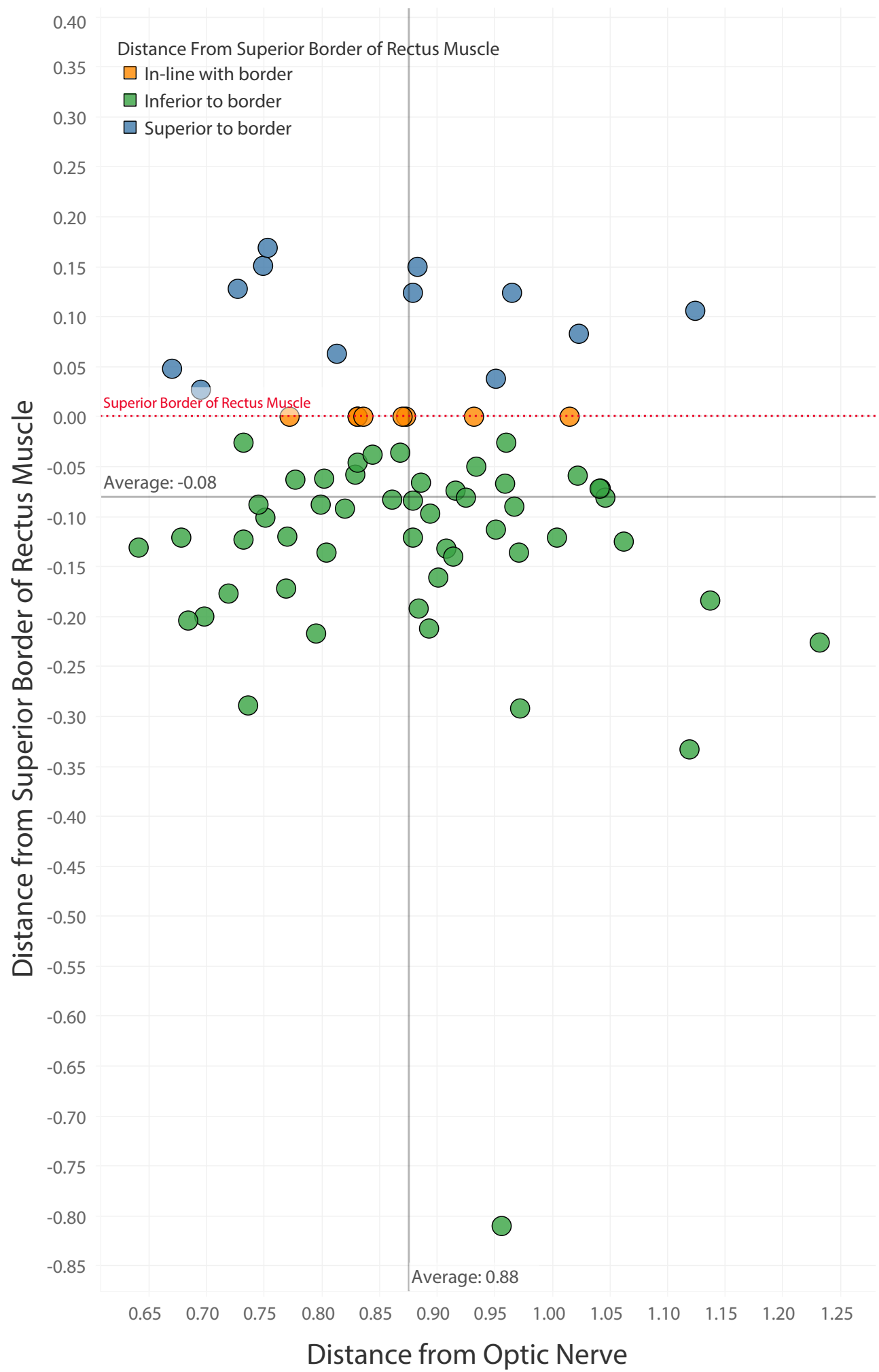

Figure 8. Penetration point of the long posterior ciliary artery. Distance from the superior border of the rectus muscle is plotted vs. the distance from the optic nerve. Data points from laterally located arteries are included $(n=71)$. 


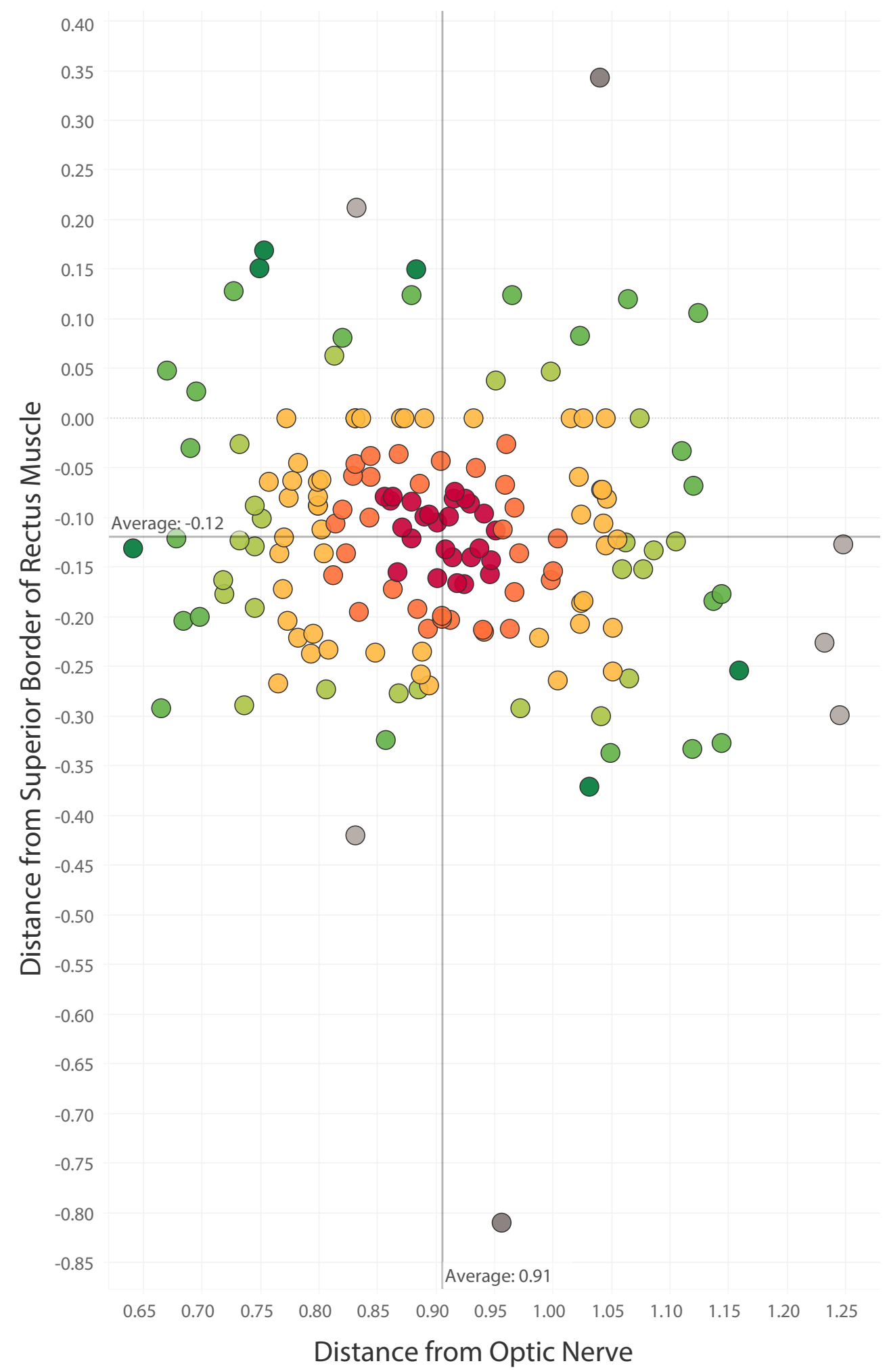

Figure 9. Surgical "danger zone". Each color change represents a $0.1 \mathrm{~cm}$ diameter increase beyond the center point of the average penetration point of the long posterior ciliary artery. Ninety-two percent of all data points fall within $0.6 \mathrm{~cm}$ diameter from the center point.

Conflict of Interest: No conflicting relationship exists for any author. 


\section{Discussion}

The purpose of this study was to evaluate variation of the long posterior artery, to create a surgical "danger zone" along its path as it pierces the posterior sclera and travels to the major arterial circle of iris, and to determine any factors that may predispose patients to anterior segment ischemia following strabismus surgery. These factors may include number of arteries, sex, medially or laterally located arteries, and differences between right and left eyes.

Disinsertion of the rectus muscles results in an interruption of blood flow to the major arterial circle of iris due to loss of blood flow through the anterior ciliary arteries found within the muscle [11]. The long posterior ciliary artery is now the sole source of blood flow supplying the anterior segment in that area. The number of long posterior ciliary arteries per eyeball varies in the literature. Some studies even found that $97 \%$ of people have both medial and lateral arteries in each eye $[9,12]$. The current study determined a much larger number of patients (33\%) only have one long posterior ciliary artery compared to two, which may predispose these patients to anterior segment ischemia. Determination of such predispositions may help guide management of these patients with more frequent postoperative monitoring and initiating treatment plans earlier post-op.

Due to the unilaterality of many ocular procedures, it is important to note differences in length between medially and laterally located long posterior ciliary arteries. Medially located arteries penetrated the sclera more distally and more inferiorly than laterally located arteries. This difference may be useful for surgeons performing operations on one side of the eye versus the other [13]. The fact that $67 \%$ of eyes had both medially and laterally located arteries, $29 \%$ had one artery located medially, and only $4 \%$ had one artery located laterally suggests that procedures involving the medial orbit/eye may increase risk of damage to the long posterior ciliary artery as they are found in higher frequency on the medial side.

The increased mean distance of long posterior ciliary artery scleral penetration from the optic nerve in eyes from male cadavers compared to female may increase risk for males undergoing invasive procedures. Procedures involving the medial, lateral, and posterior orbital walls may damage this artery due to its delayed penetration through the sclera compared to females [13-15].

Mapping a surgical "danger zone" may help surgeons avoid damage to the long posterior ciliary artery during invasive procedures or probe insertion, as many of these procedures require precise incision or device placement. Knowledge of the exact location during invasive procedures such as cryoprobe placement, external scleral buckling of the fovea, resection of choroidal melanoma, intraocular injections, orbital decompression, and other choroidal procedures may help surgeons locate the long posterior ciliary artery to avoid damage and disruption of blood flow. This knowledge may help prevent hemorrhage or other post-operative complications including ASI [15-19].

Further studies should attempt to evaluate a more diverse population and continue to elucidate variations in the long posterior ciliary artery anastomosis with the anterior ciliary arteries.

This study demonstrates that a larger percentage of patients may be predisposed to anterior segment ischemia than previously reported. It also provides a surgical "danger zone" that will enable surgeons to avoid damaging the long posterior ciliary artery during invasive choroidal and orbital procedures. This knowledge will facilitate more appropriate management of these patients including preoperative screenings, intraoperative isolation of the long posterior ciliary artery, more frequent postoperative monitoring, and accelerated initiation of treatment plans.

\section{Conflict of Interest}

No conflicting relationship exists for any author.

\section{References}

1. Bullock JD, Falter RT, Downing JE, Snyder HE. Ischemic ophthalmia secondary to an ophthalmic artery occlusion. Am J Ophthalmol. 1972;74(3):486-493.

2. Pineles SL, Chang MY, Oltra EL, et al. Anterior segment ischemia: Etiology, assessment, and management. Eye. 2018;32(2):173-178.

3. Rehman I, Mahabadi N, Ali T. Anatomy, Head and Neck, Eye Ciliary Muscles. In: StatPearls [Internet]. Treasure Island (FL): StatPearls Publishing; 2020.

4. Gupta N, Motlagh M, Singh G. Anatomy, Head and Neck, Eye Arteries. 2020 Jul 27. In: StatPearls [Internet]. Treasure Island (FL): StatPearls Publishing; 2020

5. Olver JM, Lee JP. The effects of strabismus surgery on anterior segment circulation. Eye (Lond). 1989;3 ( Pt 3):318-26.

6. Song Y, Song YJ, Ko MK. A Study of the Vascular Network of the Iris Using Flat Preparation. Korean J Ophthalmol. 2009;23(4):296.

7. Hayreh SS. Occlusion of the Posterior Ciliary Artery. Arch Ophthalmol. 2011;100(9):1481.

8. Velez FG, Davila JP, Diaz A, Corradetti G, Sarraf D, Pineles SL. Association of change in iris vessel density in optical coherence tomography angiography with anterior segment ischemia after strabismus surgery. JAMA Ophthalmol. 2018;136(9):1041-1045.

9. Barabino S, Dana MR. Posterior ciliary artery anatomy. Invest Ophthalmol Vis Sci. 2004;45:1641-1646.

10. Hills M. The Design and Analysis of Clinical Experiments. J R Stat Soc Ser A. 1987;150(4):400.

11. Lingua RW, Diamond GR. Techniques of Strabismus Surgery. In: Ophthalmology. 2009.

12. Erdogmus S, Govsa F. Topography of the posterior arteries supplying the eye and relations to the optic nerve. Acta Ophthalmol Scand. 2006;84(5):642-649.

13. Humphrey CD, Kriet JD. Surgical approaches to the orbit. Oper Tech Otolaryngol - Head Neck Surg. 2008;19(2):132-139.

14. Kennerdell JS, Maroon JC, Malton ML. Surgical approaches to orbital tumors. Clin Plast Surg. 1988;15(2):273-282.

15. Boboridis KG, Bunce C. Surgical orbital decompression for thyroid eye disease. Cochrane Database Syst Rev. 2011;(12):CD007630.

16. Birchard S, Sherding RG. Saunders Manual of Small Animal Practice.; 2006. W.B. Saunders, USA.

17. Siam AL, El-Mamoun TA, Ali MH. A restudy of the surgical anatomy of the posterior aspect of the globe: an essential topography for exact macular buckling. Retina. 2011;31(7):14051411.

18. Ryan SJ, Sadda SVR, Hinton DR, Schachat AP, Wilkinson CP, Wiedemann P. Retina (5th Edtn).; 2012. doi:10.1016/C2010-169566-1

19. Lee JP. Surgical management of nystagmus. Eye (Lond). 1988;2 ( Pt 1):44-47. 